\title{
Barriers and Facilitators to Implementation of Health System Interventions Aiming to Welcome and Protect Immigrant Patients: a Qualitative Study
}

\author{
Altaf Saadi, MD, MSC ${ }^{7}$ (D) , Uriel Sanchez Molina, B.A. ${ }^{2}$, Andrée Franco-Vasquez, B. . $^{3}$, \\ Moira Inkelas, $P h D, M P H^{4}$, and Gery W. Ryan, $P h D^{5}$
}

'Department of Neurology, Massachusetts General Hospital, Harvard Medical School, Boston, MA, USA; ${ }^{2}$ Dominican University, River Forest, IL, USA;
3David Geffen School of Medicine, University of California Los Angeles, Los Angeles, CA, USA; ${ }^{4}$ University of California Los Angeles Fielding School of
Public Health, Los Angeles, CA, USA; ${ }^{5}$ Department of Health Systems Science, Kaiser Permanente Medical School, Pasadena, CA, USA.

BACKGROUND: At the same time that federal policymakers have enforced restrictive immigration policies, healthcare systems across the USA are developing, and have implemented, interventions aimed at addressing immigration-related stressors faced by immigrant communities. Yet, little is known about the contextual determinants that influence their implementation success. Using the Consolidated Framework for Implementation Research (CFIR), this study identifies factors enabling or challenging the implementation of interventions aimed at mitigating immigration-related stressors in the healthcare context.

METHODS: We used a qualitative research design to conduct 38 semi-structured interviews with stakeholders involved in implementation of interventions at 25 healthcare facilities across 5 states with the highest undocumented immigrant populations (California, Texas, New York, Florida, and Illinois). Interviews were conducted from May through August 2018. Constant comparative analysis was used to identify barrier and facilitator themes. Deductive coding was thereafter used to categorize themes according to CFIR domain.

RESULTS: Barriers to implementation included perceptions of legal complexity and challenges to adopting such systemic strategies. Facilitators included a national policy climate that had brought immigrant health to the forefront, allowing for leveraging momentum towards institutional change; communication among healthcare personnel; existing community partnerships with immigrant rights and service organizations; and a shared sense of mission centering health equity. Local variation in immigration-related policies (e.g., local law agencies enforcing federal immigration laws) and heterogeneity of local immigrant communities also impacted implementation. Champions and informal leaders were integral to institutional efforts but not sufficient for sustainability. Perceived urgency to act superseded evaluation considerations, with all interventions in initial phases of implementation. Future iterations and evaluations of these

\footnotetext{
$\overline{\text { Prior Presentations Preliminary findings were presented in } 2019 \text { as an }}$ oral abstract presentation at the American Public Health Association Annual Meeting and at a Social Medicine Community Rounds at Cam bridge Health Alliance.

Received August 31, 2020

Accepted April 1, 2021

Published online May 13, 2021
}

interventions are needed to establish best practices and implementation determinants.

CONCLUSION: This is the first systematic study describing implementation determinants of immigration-related interventions across health systems. Identifying these determinants provides guidance to other healthcare organizations to effectively strategize and ensure implementation success.

KEY WORDS: immigrant health; implementation research; health systems; disparities.

J Gen Intern Med 36(10):3071-9

DOI: $10.1007 / \mathrm{s} 11606-021-06788-4$

(c) Society of General Internal Medicine 2021

\section{INTRODUCTION}

The Trump administration's approach to immigration was characterized by restrictive immigration policies, increased immigration enforcement, and anti-immigrant rhetoric. This political climate has been associated with adverse health outcomes among immigrants ${ }^{1}$, including dissuading immigrants from seeking health and social services. ${ }^{2}$ The negative impacts of these sociopolitical stressors transcend an immigrant's legal status. They do not only affect undocumented immigrants. They also exacerbate health disparities among immigrants with lawful authorization, US citizens in mixed immigration status households, and US citizens from the same ethnic/minority communities targeted by restrictive immigration policies due to their "perceived illegality." 1-6

Healthcare institutions across the USA implemented a wide range of systemic policies and actions to respond to this antiimmigrant climate and address their patients' needs. Our previous work identified policies and actions healthcare facilities adopted to address perceived immigration-associated risks among patients and practitioners ${ }^{7}$. In this paper, we refer to these policies and actions as "interventions." Potential interventions include the following: limiting cooperation with immigration enforcement on facility premises; not documenting immigration status in medical records; and establishing medical-legal partnerships to provide an avenue for addressing 
legal needs (further detailed in Table 1) ${ }^{7}$. Yet, little is known about the spectrum of contextual determinants that influence the success of the interventions.

Factors influencing the implementation of immigrant health-related interventions have not been well studied. Understanding these factors is crucial to guiding healthcare facilities in creating their own intervention variations and scaling interventions to address patient needs and optimize healthcare for all patients, regardless of immigration status ${ }^{8-12}$. This study uses the Consolidated Framework for Implementation Science (CFIR) ${ }^{10}$ to identify factors enabling or challenging the implementation of interventions aimed at mitigating immigration-related stressors in the healthcare context.

Table 1 Health Care Facility Interventions Aiming to Address Immigration-Related Stressors

\begin{tabular}{ll}
\hline \hline Intervention target & $\begin{array}{l}\text { Sample policies and } \\
\text { actions }\end{array}$
\end{tabular}

Risk of immigration enforcement at or near facilities

Risk of disclosure of patient immigration status

Risks associated with patient-level stressors (e.g., fear of deportation, fear of stressors jeopardizing immigration status, internalized feelings of shame, deservingness, and disempowerment regarding immigration status)

Practitioner-level stressors (e.g., direct impact from immigration policies, uncertainty about response to immigrant patients' needs)

Coordination of interventions
Legal

- Limiting cooperation with immigration enforcement

- Designating public vs. private spaces where patient care is directly provided

- Initiating alternative models for delivering healthcare services (i.e., telehealth)

- Limiting the collection and documentation of immigration status in medical records - Ensuring protection and confidentiality of medical records

- Providing alternative payment methods (i.e. out-of-pocket payment options)

- Seeking medical-legal collaborations to meet the legal needs of immigrants - Educating patients regarding their legal rights preparedness within larger emergency preparedness

- Affirming care $\begin{array}{ll}\text { Patient } & - \text { Affirming } \\ \text { resiliency } & \text { messages }\end{array}$

- Seeking avenues to nurture empowerment and engagement among immigrants (i.e., advocacy skills workshops, media and story-telling skill-building programs, voter registration)

- Providing supportive services for employees who are immigrants

- Training clinicians on providing healthcare to immigrants

- Appointing an immigration point person or task force
- Combining deportation

\section{METHODS}

\section{Study Design and Sample}

We used a qualitative research design involving a 3-stage sampling frame. First, we selected the 5 states with the largest undocumented immigrant populations: California, Texas, New York, Florida, and Illinois ${ }^{13,14}$. We focused on states with the largest undocumented immigrant populations because they are disproportionately impacted by anti-immigrant policy climates; consequently, healthcare facilities would more likely be implementing interventions to mitigate immigration-related stressors. Second, we used informational interviews with local immigrant rights leaders to identify facilities in each state that were implementing such interventions. The first author (A.S.) identified these leaders using pre-existing community partnerships established during prior immigration-advocacy efforts. We did not target specific types of healthcare facilities as our goal was to capture the range and scope of interventions regardless of institution type. Third, we identified and recruited stakeholders at each facility who were involved in the implementation process. We used both purposeful and snowball sampling. All participants provided verbal informed consent. The University of California Los Angeles IRB approved this study.

\section{Data Collection}

We used a semi-structured interview guide (Appendix) informed by the literature, CFIR domains ${ }^{10}$, and community partner input to conduct in-person or telephonic interviews between May and August 2018. We stopped conducting interviews when we observed thematic saturation. Two individuals declined to participate. All but one interview was audiorecorded. Interviews were professionally transcribed. The study results are reported in compliance with the Standards for Reporting Qualitative Research (SRQR) guidelines.

\section{Data Analysis}

We used constant comparative analysis to identify implementation barriers and facilitators. Authors A.S. and G.R. met weekly during the interview process to discuss emerging themes. Later, authors A.S. and U.S.M. used a deductive coding strategy to categorize identified barriers and facilitators by CFIR domain. We used discussion and consensus between authors A.S., U.S.M., and G.R. to resolve coding discrepancies. We used Dedoose (Version 8.0.42) qualitative data management software to facilitate analysis.

\section{RESULTS}

We conducted 38 interviews across 25 facilities. Interviews included Federally Qualified Health Centers (FQHCs) $(n=13)$, academic and private hospitals $(n=7)$, and county facilities $(n=5)$. Participants included providers in clinical and/or 
administrative roles $(n=27)$ and senior executives $(n=11)$. Table 2 summarizes study sample characteristics. Themes with illustrative quotes organized by CFIR domains are listed in Table 3

\section{Intervention Characteristics}

Entanglement with Law Enforcement and Perceptions of Legal Complexity. Although interventions did not target undocumented immigrants (see Table 1), the perceptions of legal challenges associated with immigration status and potential institutional entanglement with law enforcement were key barriers to implementation. Many participants explained that previous institutional policies were insufficient to address heightened fears. A Texas physician described: "when we looked at the [hospital] policy, the policies address law enforcement and they give a list of every possible law enforcement including delegates of ICE, just saying that we need to do what they need us to do. They're trying to have policies that are as generic as possible but that's not always appropriate." Thus, new policies were created to address these gaps, including limiting cooperation with ICE when there were conflicting goals impeding patient care (e.g., use of shackles, expedited discharge, or inappropriate discharge to detention).

Many healthcare personnel "were terrified" of these interactions, particularly immigrant staff or those in mixed immigration status households because "many of our staff are from the communities we serve." A Texas physician described a colleague's anxiety when interfacing with immigration enforcement at a detained patient's bedside: "he's a citizen but his partner is not... He was really nervous every time [immigration agents] were taking his name because he was terrified about the implications for his partner." Interventions therefore needed to be firmly grounded in the law and accompanied by adequate training of staff to offer scared providers sufficient reassurance.

Some participants described the time required to vet immigration-related interventions by institutional legal personnel to protect against the possibility of lawsuits as a barrier, with immigration perceived as a "hot potato" issue undergoing extra scrutiny. Not only did this process delay intervention implementation, but it also sometimes resulted in inadequate policies that prioritized litigation concerns over clinician needs. When discussing a policy pertaining to immigrant detainees, a Texas physician relayed: "It turned out that the doctors can write an order for removing or partially releasing the shackles. But an order to who? You can put an order in but it's up to the guards to decide. I know what it is, it's more covering yourself that you tried to do something."

\section{Outer Setting}

Local Policy Contexts. Differences in national, state, and local policies impacted participants' ability to address patient needs and provide optimal patient care. The outer setting mediated the perceived importance of immigrant health, presence (or absence) of restrictive immigration policies, and local

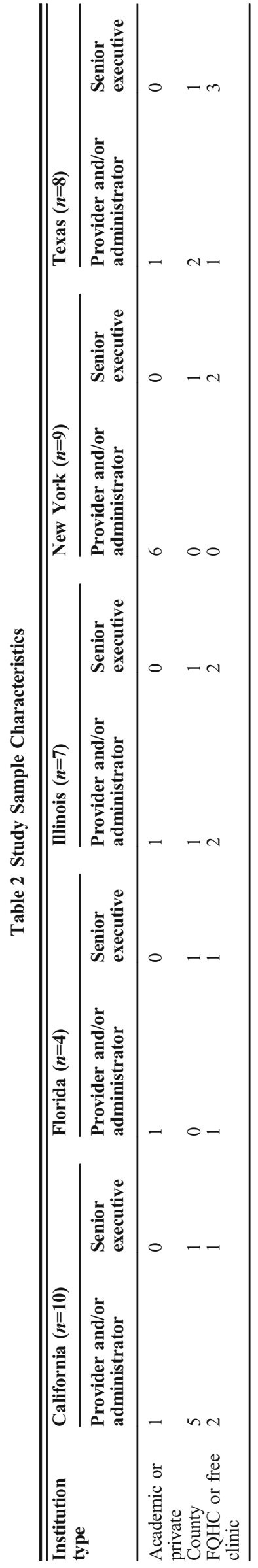


Table 3 Summary of Themes and Illustrative Quotes across CFIR Domains

\begin{tabular}{ll}
\hline \hline CFIR domain & Theme \\
\hline $\begin{array}{l}\text { Intervention } \\
\text { characteristics }\end{array}$ & $\begin{array}{l}\text { Entanglement with } \\
\text { law enforcement and } \\
\text { perceptions of legal } \\
\text { complexity }\end{array}$ \\
\end{tabular}

Illustrative quotes

- "The ICE agents were awful... They wouldn't allow the team to even have private conversations with the patient. There was no patient-doctor confidentiality at all. It was really awful, so... I was like, "We have to push administration [for a policy]."

-"That's what I would say we needed more training on because ... is the power of attorneys even real? Do we need to notify them? Is there a different form? What do you do when ICE comes to our clinic? Do we get arrested? Can we close our doors? We didn't

know none of that stuff... we didn't know legally how far we can push. Or what type of representation we might need if we denied access to whoever."

- "Giving people wrong information is not serving them well. So we talked to our legal [department]...who really gave us quite a bit of excellent

information."

Local policy contexts (across community variation)

Heterogeneous immigrant communities (within community variation)
-"We have to consider our actions within the larger county [Miami]. We don't want a red target on the organization."

- "LA has always been the city of immigrants. We've always had the immigrant population push for more progressive policies within the city. Developing these particular processes for immigrant patients becomes a lot easier because the resources are there."

-"It's been really difficult to get anything going in any organized fashion in Houston. I think that people are trying to do the right thing but they don't want to be very visible doing it"

-"We have some very unique immigrant populations. You know, with Cubans and Haitians... They're very different.' about how they're
Table 3. (continued)

\begin{tabular}{lll}
\hline \hline CFIR domain & Theme & Illustrative quotes \\
\hline Inner setting & Communication and & "The big thing is that
\end{tabular}

trust among

" The big thing is that

healthcare personnel

-We have a lot of meetings, and at our leadership team meeting this [immigration] was really a common topic, just communication at all these different venues."

-"The overarching discussion point [at staff meetings] was finding out what the fears were... there's a level of trust, knowing that we're here for them. It was the constant reassurance on all levels, management, providers, staff... that we were here to serve their needs. We would help them in any capacity we could."

Inside change community partnerships

Institutional mission around health equity

"We're a partnership so they were pretty in touch with a lot of what was going on the policy side, we were doing a lot of swapping things, a lot of checking in with them." ."It's important that] partnerships with community-based organizations and legal help organizations are strong and we work through them to educate the community and have the community educate the facilities, so there are some eyes and ears on the ground getting constant feedback in both directions."

- "That's why I think the success of health centers like ours comes down to that we don't make it a Republican thing. We don't make it a

Democrat thing. We don't make it whether you're U.S. citizen or not a U.S. citizen. We always put, "Is this patient healthy?" "Are we keeping the patient healthy enough?" "Is he or she an active member of his or her community?"

- Just with having that type of mission already, that kind of changes how we even treat anyone walking through our doors. I mean, you literally could be a guy from Mars, and if you do not have access to healthcare, we'll give you access to healthcare." 
Table 3. (continued)

\begin{tabular}{ll}
\hline \hline CFIR domain & Theme \\
\hline $\begin{array}{l}\text { Characteristics of } \\
\text { individuals }\end{array}$ & $\begin{array}{l}\text { Champions, } \\
\text { advocates, and } \\
\text { informal leaders }\end{array}$
\end{tabular}

Illustrative quotes

•"There are pockets of physicians who are really attuned to this new environment and what are patient rights.... It's all within pockets of physicians who are very active."” "I have a provider here who literally was like, "I'll get arrested first before I let my patient go." So I'm like, "Okay, so if this doctor gets arrested, do we have bail? Do we have a lawyer on call to represent him to get him out of bail? Can he lose his license?" So we had to get in-house counsel on that."

-"Identifying a champion in the leadership is probably the best way. Like someone who's going advocate for you with others in the leadership. Someone that people already are used to saying yes to, and they know politically who to ask for what and how to get people to say yes." "We really haven't [done evaluations] and it still feels pretty reactive right now."

-"We're not asking our patients, "Are you coming here because you're undocumented, or because you're a refugee, and seeking asylum?

-"[We've received] feedback of the organizations that we work with, so we have been recognized many times by these community organizations and advocacy agencies. But I mean that's not a real scientific measurement of it." resources for immigrant patients across the immigration status spectrum.

The changing national policy climate brought immigrant health to the forefront, prompting clinicians and senior executives to be more vocal about immigrant health. As one Texan physician described: "maybe it's because the issues are more pressing, [or] maybe it's because they feel more empowered to speak up, but more people now than before are willing to sound an alarm." This increased attention led to newfound awareness among personnel, greater conversations, and building momentum regarding mitigating immigrationassociated risks in the healthcare context.

Variation in "immigrant-friendly" state or city policies influenced implementation. Participants in California, for example, discussed state bill SB54 that prevents local law agencies from enforcing federal immigration laws. Consequently, it was easier for them to adopt similar institutional policies by using state policy as a guide and leverage for change. Participants described how public policies relating to obtaining driver's licenses or identification cards increased undocumented immigrants' perceived risks to accessing healthcare. An administrator at a Florida FQHC noted, "people get picked up driving without a license because unlike a lot of states, Florida doesn't allow for undocumented folks to get a driver's license"; conversely, in California, where all immigrants can obtain a driver's license, transportation barriers were less salient. Health system interventions could not overcome patient fears consequent to such state and local policies.

Nuances beyond immigration policy impacted implementation. For instance, a senior executive in Florida discussed how active shooter preparedness superseded and delayed immigration-related institutional efforts. Participants in Texas and Florida shared that identifying local services open to individuals regardless of immigration status was part of their institutional hurricane preparedness planning.

Local climates also influenced other resources available for immigrants. One administrator at a Los Angeles FQHC explained: "LA has always been the city of immigrants... Developing these particular processes for immigrant patients becomes a lot easier because the resources are there." Furthermore, this climate influenced how facilities went about their immigration-related efforts. In more conservative states, "powerful institutions don't want to be public about how they're helping undocumented immigrants, even if they are." These restrictive immigration local policy climates made it challenging for healthcare facilities to identify and advertise as immigrant-friendly spaces due to fear of facing backlash.

Heterogeneous Immigrant Communities. Local variations within immigrant populations influenced the conversation about immigration-related efforts and were key considerations during intervention design to address the unique needs of each community. For example, in Florida, Cuban immigrants who enjoyed preferential treatment in US immigration law and were given a swift, direct path to citizenship did not require the same interventions like medical-legal partnerships as did immigrants from Central America. In Chicago, the large population of African and Polish immigrants needed to be included in community-based immigrant efforts, requiring material in languages other than Spanish.

\section{Inner Setting}

Trust and Communication among Healthcare Personnel. Implementation success was contingent around existing cultures of communication ("communication has always been very key"), 
including who to include in communication and how to communicate (i.e., quality of communication, including trust).

Participants emphasized including diverse roles across the healthcare system ("the big thing is that we don't work in silos") because multiple healthcare professionals interfaced with immigration-related issues pertaining to patients. Implementation failures occurred when conversations were physician-dominated and excluded nurses or other staff like hospital security. One physician at a Texas hospital described how physicians had delineated a plan about interfacing with immigration officials accompanying patients from immigration detention. However, when one such incident occurred, the nurse did not notify the medical team according to plan. This was partially attributed to not having nursing representation during planning discussions. Legal teams were also identified as critical to include to avoid potential legal ramifications: "we really need some legal backing and the legal people saying, 'Yes, we'll support you if this happens.'... I think to do [educational sessions for clinicians] without [this support] is doing a disservice because we're telling them to do something that they could get fired for."

Cultures of trust and open communication across the medical hierarchy were key elements to the quality of communication, facilitating exchange of ideas around a politicized and personal topic (immigration) and enabling iterative feedback to optimize implementation. These cultures of trust were facilitated by smaller staff sizes; existing mechanisms for continuous communication (i.e., regular all-staff meetings or designated health equity committees); and support from senior executive leadership. A participant stated: "When you're in a smaller organization, you really do have that kind of closeness" and "what really made us take action was the concern that was coming from our teams."

Timeline to implementation was longer in facilities without existing mechanisms of communication. A participant from a New York community hospital described their first point of action as convening an "Immigrant Health Working Group" to establish regular discussion about immigration-related challenges, before other interventions could be implemented. At a county facility in Texas, a physician lamented the absence of a central mechanism for addressing immigration-related issues; she described approaching various hospital leaders to identify answers to immigration-related questions (e.g., inpatient director, outpatient director, security office, risk management, and ethics committee).

\section{Inside Change Dependent on Outside Community} Partnerships. Partnerships with immigrant-serving community-based organizations were integral to successful implementation efforts. These partnerships facilitated the adaptation, augmentation, and rapid release of resource guides, Know Your Rights information, and intervention development and dissemination. Thus, there was an increase in both scale and number of interventions among facilities with pre-existing community partnerships. One FQHC in New York leveraged an existing partnership with local police to host a community event to assuage immigrant patients' fears about police cooperation with immigration enforcement. One administrator at an FQHC in Illinois said: "Being noticed in the community... well, that's one of the practices that was very easy for us to do because we were already there."

Community partnerships also provided institutions continuous feedback regarding the community's needs. As one administrator at a FQHC in California explained: "we haven't missed a beat because we understand the need of continuous education in community." At other healthcare facilities, absence of community partnerships represented a fundamental barrier associated with fewer implemented interventions and slower responses. If interventions were implemented, they did not evolve in an iterative fashion with community feedback ("Our biggest challenge was getting stuck").

Institutional Mission Around Health Equity. A shared sense of institutional mission around health equity was common among facilities successful in implementing interventions mitigating immigration-related stressors. Many participants even began by first sharing their institutional history before describing their implementation efforts. An administrator at an academic-community hospital in Illinois said: "That's the very beginning of everything but it's important to mention because this hospital was built and created for the use of immigrants. Because of that, we are very immigrant-friendly and it's something that we try to reflect in our services on a daily basis." This sense of mission extended to healthcare personnel: "this is a hospital for indigent patients. So the nurses who work here want to work here for that reason. Same with the doctors. That's what the community is here." This was particularly true for FQHCs where institution, senior executive, and healthcare personnel missions often aligned around "thinking for and by our patients." In this light, even financial or other limited resources were not deemed as significant as the driving mission.

Healthcare facilities that faced challenges often grappled with discrepant beliefs about the institution's mission, which was more likely at large academic medical centers or county facilities. Mission discrepancies involved competing objectives like patient care, profit generation, research production, or maintenance of medical prestige. The CEO at one county facility described the financial tension as such: "If I have to carve out of my limited resources the ability to do legal counseling, social, emotional, family-based trauma counseling, okay. But let's say that that costs me $\$ 50$ million. Well, then I'm not going to be able to do $\$ 50$ million of clinical care." Competing missions made institution-backed interventions difficult, with providers caught in between multiple, competing hierarchies, and not optimally positioned to meet their patients' needs. 


\section{Characteristics of Individuals}

Champions, Advocates, and Informal Leaders. Individual healthcare personnel who organized efforts both within and outside their institutions were essential to intervention implementation. Efforts included convening local healthcare personnel to exchange best practices or providing training to raise awareness and urgency around immigration-related issues. Implementation success was more likely when these individuals' efforts had institutional and senior leadership support. A Texas county clinician described: "there are pockets of physicians who are really attuned to this new environment and what are patient rights.... It's not the hospital administration that will say, 'You need to go to this training.' It's all within pockets of physicians who are very active."

Various causes motivated individual advocates. Several participants reported that "many of us are either immigrants or the children of immigrants" while others described deeply held beliefs about their roles extending beyond clinic walls. At one free clinic in Illinois, a clinician-administrator said: "We wear many hats here, so people have known me as their clinician. But I help them with sick appointments, I help them with food pantries, I've interpreted, I may have gotten [them] a T-shirt at a garage sale. They see us all the time, they see us everywhere." These committed individuals were critical for fostering a culture of bottom-up change that facilitated intervention implementation.

However, it was insufficient to rely solely on these individual advocates. Some participants described how "there's a lot of people that don't necessarily have the bandwidth to be pushing these things forward" without institutional support. One local champion in Los Angeles said: "there's room for innovation in this space and someone needs to be charged with or be given the freedom to be able to do that." At one facility, all efforts halted when the motivated individual went on maternity leave. When these efforts were led by ad hoc healthcare personnel, there was less time devoted to them, more disagreements, and fewer interventions implemented.

\section{Implementation Process}

Initial Phases of Implementation Without Measurement or Evaluation Mechanisms. Many interventions were in the initial phases of implementation and most participants expressed that the perceived urgency to act superseded evaluation considerations. Participants underwent rapid informal quality improvement iterations. A New York clinician involved in implementing a deportation preparedness plan for immigrant parents discussed how "initially we had paper holders with these brightly colored papers right next to the clerks where they register... and they weren't really going. We thought, 'Maybe people feel a little self-conscious taking the paper.' So, we put it in a different part of the waiting room where they could look at it while sitting."

Many participants acknowledged difficulty evaluating outcomes when they did not have enough resources to conduct formal assessments and did not capture immigration status in medical records. Some worried about potential unintended consequences to implemented interventions, thereby emphasizing the need for future formal assessments. A CEO in Illinois expressed: "We're actually concerned from some people who have talked to us that if we put [safe space] signage up, it'll have the opposite effect, that it'll scare people, because they'll think, 'well, the cops have seen those signs. Or the ICE agents have seen those signs,' and they'll know that this [facility] is a place that provides services to the undocumented."

\section{DISCUSSION}

Healthcare systems are adaptive systems that respond to evolving patient and provider needs, including shifting immigration policies contributing to heightened anxieties altering healthcare-seeking behaviors and engagement. Our study finds several factors that facilitated system-level responses to immigration-related stressors.

One integral factor is strengthening and developing effective community partnerships. Traditionally, community partnerships focus on addressing patients' economic, environmental, and social needs outside the healthcare system ${ }^{15}$. Community partnerships can also facilitate patients' entry into the healthcare system (e.g., via clinical care or research studies) while maintaining a separation between "community" and "health system" spaces. Our study advocates for community-institutional relationships permitting coordination of efforts outside of the health systems' boundaries. This study also recognizes community organizations' expertise as a critical resource needed to create innovative strategies to address emerging patient needs within healthcare institutions. Without these community partnerships, our participants described health systems as unable to effectively respond to immigrant patient and provider needs at an organizational level. As community-institutional partnerships can take time to develop and maintain ${ }^{16}$, it may be beneficial for institutions to proactively establish these partnerships to ensure timely responses to public policies targeting marginalized populations.

Our research further highlights the importance of robust networks that cross administrator, clinician, staff, and patient levels within and outside a healthcare facility. Developing and enhancing these networks is an important and viable implementation strategy ${ }^{12}$. Healthcare facilities should therefore consider creating structured networking opportunities (e.g., service provider resource fairs for patients, or trainings increasing personnel familiarity with community-based services).

Our findings underscore that alignment of organizational mission and strategic priorities is essential for successful implementation of interventions aiming to protect and 
welcome immigrant patients and providers. Literature on patient quality and safety corroborates this: a lack of a cohesive mission, conflicts between multiple organizational missions, and poorly defined goals are common obstacles faced by organizations struggling to improve quality ${ }^{15,17}$.

Operational infrastructures to address health equity issues - including decision-making pathways, program development support, and resource allocation-facilitated health facilities' response to immigration-related stressors. By using existing infrastructures, institutions were able to stay nimble in their response to evolving policy changes targeting vulnerable population subgroups like immigrants. This is akin to disaster response frameworks that consider mitigation and preparedness as essential components to response ${ }^{18}$. Our study posits that ramifications of public policies can be viewed similarly as "disasters" for patients and providers, and response to immigration policies can therefore use a similar approach to mitigation, preparedness, response, and recovery ${ }^{18}$.

Conflicts between organizational missions have been raised most extensively in discussions of the threepronged missions of Academic Health Centers (AHCs): (1) patient care, (2) graduate medical education, and (3) research ${ }^{19-21}$. Providers are caught within power structures responding to each mission, hindering their ability to provide optimal patient care. Despite a growing emphasis among AHCs to address health disparities, there is insufficient empirical evidence showing how this is being operationalized ${ }^{22,23}$. Fewer mission discrepancies may explain why $\mathrm{FQHCs}$ were more successful in implementation efforts in our sample. However, continued research on evidence-based strategies to achieve equity goals across healthcare systems is needed.

Individuals with personal exposure to the impact of immigration policies significantly contributed to the design and implementation of interventions mitigating immigration-related stressors. A diverse healthcare workforce inclusive of immigrants should therefore be heeded as an important ingredient to meeting the needs of immigrant communities and bottom-up organizational change. Previous studies have enumerated the benefits of racial and ethnic diversity of the healthcare workforce ${ }^{24}$.

But diverse workforce members are insufficient without programs and groups supporting diversity. Without organizational support structures, providers in our study described increased burnout, concern about not meeting their patients' needs, and personal anxieties about how they themselves were impacted by anti-immigrant policies. Support took the form of reassurance from management, legal support for immigration procedures, and employee assistance programs (e.g., counseling). Diverse organizational cultures receptive to change expedite implementation of institutional policies when patient needs are acutely stressed, as in the case of immigrants during the Trump administration.
Additionally, our findings build upon the growing literature on innovative strategies used by healthcare systems to respond to patients' social needs with the goal of advancing health equity. Whether it is providing fruit and vegetable prescriptions for patients experiencing food insecurity ${ }^{25}$, fostering lesbian, gay, bisexual, transgender, and queer (LGBTQ)-friendly services ${ }^{26}$, or adopting immigrant-friendly strategies ${ }^{7}$, it is necessary for healthcare facilities to consider the unique challenges patients experience and serve as places of innovation to better address the needs of communities they serve. Facilities in our sample adopted interventions to protect and welcome immigrant patients without an evidence base, necessitating future iterations to establish best practices.

We found CFIR an effective lens for understanding factors influencing the design and implementation of interventions without a more established evidence base. Of all the CFIR domains, the implementation process domain was mentioned least, likely because we interviewed healthcare facilities that represent the "innovators" and "early adopters" as identified by Rogers' diffusion of innovations. The barriers and facilitators we identified will likely evolve as these interventions are further developed, evaluated, and disseminated, and confront evolving crises like the COVID-19 pandemic.

Several study limitations warrant consideration. First, our investigation lacks patient perspectives. Patient perspectives are critical in understanding the effectiveness of any healthcare intervention, and this is the focus of our forthcoming research. Second, our sample does not include experiences of healthcare organizations in rural settings. Although this was outside the purview of our study question, several stakeholders alluded to potential differences in rural areas. This study should be replicated to elucidate unique considerations of implementation in rural settings. Third, although CFIR was a helpful guide for data collection and organization, the rigidity of some domains limited the appreciation of the dynamism between the environment, institutional infrastructure, intervention team, and behavior of the population targeted by these interventions. Future studies may consider other analytic frameworks such as the Baldrige Excellence Framework for healthcare ${ }^{27}$ or the Model for Understanding Success in Quality (MUSIQ) ${ }^{11}$. Strengths of this study remain the inclusion of diverse perspectives, including senior-level executives and healthcare personnel across five states with different political leanings. The COVID-19 pandemic has spotlighted immigrant health inequities, and the public health ramifications of their fears limiting health-seeking further underscore the need for future research on implementation determinants of immigration-related interventions across health systems. 


\section{CONCLUSION}

This is the first systematic study of barriers and facilitators to implementation of interventions aiming to mitigate immigration-related stressors in the healthcare context. It identifies implementation challenges and promotes development of implementation strategies in an understudied area. Elucidating implementation determinants enables other healthcare organizations considering implementing similar interventions to strategize more effectively to ensure implementation success.

Supplementary Information The online version contains supple mentary material available at https://doi.org/10.1007/s11606-02106788-4.

Corresponding Author: Altaf Saadi, MD, MSc; Department of Neurology, Massachusetts General Hospital, Harvard Medical School, Boston, MA, USA (e-mail: asaadi@mgh.harvard.edu).

Author Contribution Grace Kim, MD, and Robert H. Brook, MD, of the University of California, Los Angeles, provided assistance and feedback during the early stages of the study design. Sarah Dar, MPH, Health Policy Manager of the California Immigration Policy Center, and Sameer Ahmed, JD, of the Harvard Immigration and Refugee Clinic at Harvard Law School, provided feedback throughout the study and recommended key individuals and stakeholders to interview.

Funding Funding for this study was provided in part by grant CPAC69085_16_05 from the California Initiative for Health Equity \& Action, a statewide health equity research translation center of the University of California.

\section{Declarations:}

Conflict of Interest: The authors declare that they do not have a conflict of interest.

\section{REFERENCES}

1. Gemmill A, Catalano R, Casey JA, et al. Association of Preterm Births Among US Latina Women With the 2016 Presidential Election. JAMA Netw Open. 2019;2(7):e197084.https://doi.org/10.1001/jamanetworkopen.2019.7084

2. Page KR, Polk S. Chilling Effect? Post-Election Health Care Use by Undocumented and Mixed-Status Families. New England Journal of Medicine. 2017;376(12):e20.https://doi.org/10.1056/NEJMp1700829

3. White K, Yeager VA, Menachemi N, Scarinci IC. Impact of Alabama's Immigration Law on Access to Health Care Among Latina Immigrants and Children: Implications for National Reform. Am J Public Health. 2014;104(3):397-405.https://doi.org/10.2105/AJPH.2013.301560

4. White K, Blackburn J, Manzella B, Welty E, Menachemi N. Changes in use of county public health services following implementation of Alabama's immigration law. J Health Care Poor Underserved. 2014;25(4): 1844-1852.https://doi.org/10.1353/hpu.2014.0194

5. Pedraza FI, Nichols VC, LeBrón AMW. Cautious Citizenship: The Deterring Effect of Immigration Issue Salience on Health Care Use and Bureaucratic Interactions among Latino US Citizens. J Health Polit Policy Law. 2017;42(5):925-960.https://doi.org/10.1215/03616878-3940486

6. Morey BN. Mechanisms by Which Anti-Immigrant Stigma Exacerbates Racial/ Ethnic Health Disparities. American journal of public health. 2018;:e1-e4.

7. Saadi A, Molina US, Franco-Vasquez A, Inkelas M, Ryan GW. Assessment of Perspectives on Health Care System Efforts to Mitigate Perceived Risks Among Immigrants in the United States: A Qualitative Study. JAMA Netw Open. 2020;3(4):e203028.https://doi.org/10.1001/jamanetworkopen.2020.3028

8. Glasgow RE, Vogt TM, Boles SM. Evaluating the public health impact of health promotion interventions: the RE-AIM framework. Am $J$ Public Health. 1999;89(9):1322-1327.https://doi.org/10.2105/ajph.89.9.1322
9. Rycroft-Malone J. The PARIHS framework-a framework for guiding the implementation of evidence-based practice. $J$ Nurs Care Bual. 2004;19(4):297-304.https://doi.org/10.1097/00001786-200410000-00002

10. Damschroder LJ, Aron DC, Keith RE, Kirsh SR, Alexander JA, Lowery JC. Fostering implementation of health services research findings into practice: a consolidated framework for advancing implementation science. Implementation Science. 2009;4(1):50.https://doi.org/10.1186/1748-5908-4-50

11. Kaplan HC, Provost LP, Froehle CM, Margolis PA. The Model for Understanding Success in Quality (MUSIQ): building a theory of context in healthcare quality improvement. BMJ Qual Saf. 2012;21(1):1320.https://doi.org/10.1136/bmjqs-2011-000010

12. Powell BJ, Waltz TJ, Chinman MJ, et al. A refined compilation of implementation strategies: results from the Expert Recommendations for Implementing Change (ERIC) project. Implementation Science. 2015;10(1):21.https://doi.org/10.1186/s13012-015-0209-1

13. Migration Policy Institute. U.S. Immigrant Population by State and County. Published February 4, 2014. . https://www.migrationpolicy.org/programs/ data-hub/charts/us-immigrant-population-state-and-county

14. Pew Research Center's Hispanic Trends Project. U.S. unauthorized immigrant population estimates by state, 2016. Published February 5, 2019. https://www.pewresearch.org/hispanic/interactives/u-s-unauthorized-immigrants-by-state/

15. Gottlieb L, Fichtenberg C, Alderwick H, Adler N. Social Determinants of Health: What's a Healthcare System to Do? J Healthc Manag. 2019;64(4):243-257.https://doi.org/10.1097/JHM-D-18-00160

16. Seifer SD. Building and Sustaining Community-Institutional Partnerships for Prevention Research: Findings from a National Collaborative. J Urban Health. 2006;83(6):989-1003.https://doi.org/10.1007/s11524-006-9113-y

17. Vaughn VM, Saint S, Krein SL, et al. Characteristics of healthcare organisations struggling to improve quality: results from a systematic review of qualitative studies. BMJ Qual Saf. 2019;28(1):74-84.https:// doi.org/10.1136/bmjqs-2017-007573

18. Rose DA, Murthy S, Brooks J, Bryant J. The Evolution of Public Health Emergency Management as a Field of Practice. Am J Public Health. 2017;107(S2):S126-S133.https://doi.org/10.2105/AJPH.2017.303947

19. Konstam MA, Hill JA, Kovacs RJ, et al. The Academic Medical System: Reinvention to Survive the Revolution in Health Care. $J$ Am Coll Cardiol. 2017;69(10):1305-1312.https://doi.org/10.1016/j. jacc.2016.12.024

20. Lale A, Moloney R, Alexander GC. Academic medical centers and underserved communities: modern complexities of an enduring relationship. J Natl Med Assoc. 2010;102(7):605-613.https://doi.org/10.1016/ s0027-9684(15)30638-6

21. Conway SJ, Berkowitz SA. Population Health and Academic Medical Centers: High Cost Meets High Efficiency. Prim Care. 2019;46(4):631640.https://doi.org/10.1016/j.pop.2019.07.010

22. Edelman Alexandra, Taylor Judy, Ovseiko Pavel V., Topp Stephanie M. The role of academic health centres in improving health equity: a systematic review. Journal of Health Organization and Management. 2018;32(2):279-297.https://doi.org/10.1108/JHOM-092017-0255

23. Park B, Frank B, Likumahuwa-Ackman S, et al. Health Equity and the Tripartite Mission: Moving From Academic Health Centers to AcademicCommunity Health Systems. Academic Medicine. 2019;94(9). https:// journals.lww.com/academicmedicine/Fulltext/2019/09000/Health_Equity_and_the_Tripartite_Mission_Moving.12.aspx

24. Cohen JJ, Gabriel BA, Terrell C. The Case For Diversity In The Health Care Workforce. Health Affairs. 2002;21(5):90-102.https://doi.org/10. 1377 /hlthaff.21.5.90

25. Saxe-Custack A, LaChance J, Hanna-Attisha M, Ceja T. Fruit and Vegetable Prescriptions for Pediatric Patients Living in Flint, Michigan: A Cross-Sectional Study of Food Security and Dietary Patterns at Baseline. Nutrients. 2019;11(6).https://doi.org/10.3390/nu11061423

26. Wilkerson JM, Rybicki S, Barber CA, Smolenski DJ. Creating a Culturally Competent Clinical Environment for LGBT Patients. Journal of Gay \& Lesbian Social Services. 2011;23(3):376-394.https://doi.org/10.1080/ 10538720.2011 .589254

27. National Institute of Standards and Technology. Baldrige Excellence Framework (Health Care). NIST. Published January 27, 2010. . https:// www.nist.gov/baldrige/publications/baldrige-excellence-framework/ health-care

Publisher's Note: Springer Nature remains neutral with regard to jurisdictional claims in published maps and institutional affiliations. 